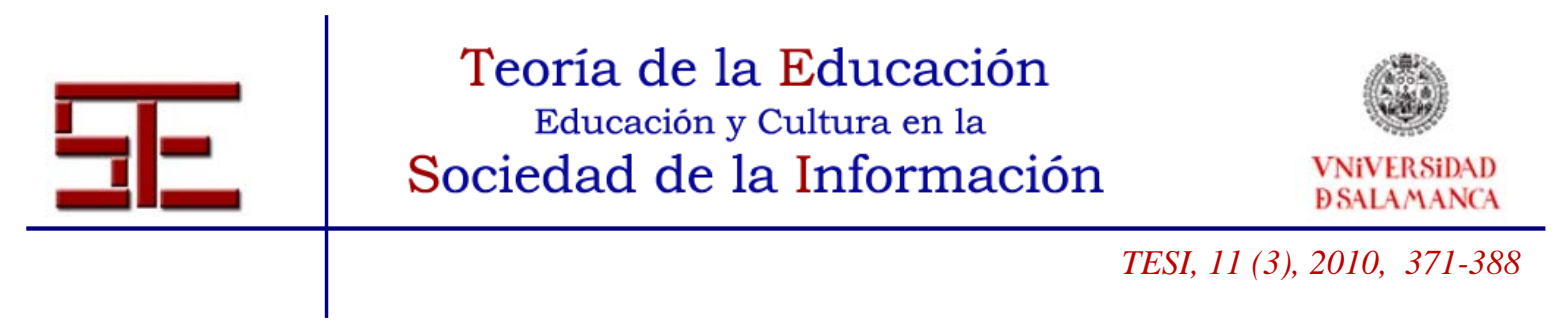

\title{
LA FORMACIÓN EN TECNOLOGÍAS DE LA INFORMACIÓN Y DE LAS COMUNICACIONES (TIC) EN LA TITULACIÓN DE TURISMO: ¿ES ADECUADA?
}

Resumen:

Este trabajo profundiza en la detección del grado de ajuste entre los conocimientos adquiridos en materia de TIC y los requerimientos demandados por las empresas del sector turístico a sus futuros profesionales. Los alumnos que acceden a la titulación de Turismo están familiarizados con el uso de las TIC pero no tienen los conocimientos suficientes para un adecuado manejo en un ámbito profesional. Por otro lado, la realidad del ámbito de la formación superior en estudios turísticos refleja una aparente necesidad de un mayor número de créditos que abarquen estos conocimientos y de un mayor número de plazas para cursarlos. Se realiza un estudio empírico en tres fases a los alumnos que realizan el practicum y a las empresas en que lo realizan. Los resultados apoyan la necesidad de optimizar los contenidos de las asignaturas genéricas de informática, contemplando las necesidades específicas de la empresa. A través de la interpretación de los datos obtenidos se establecen una serie de recomendaciones que pueden servir de apoyo a los docentes para optimizar el trabajo en el aula.

Palabras clave:

Competencias TIC, titulación de Turismo, practicum, Espacio Europeo de Educación Superior

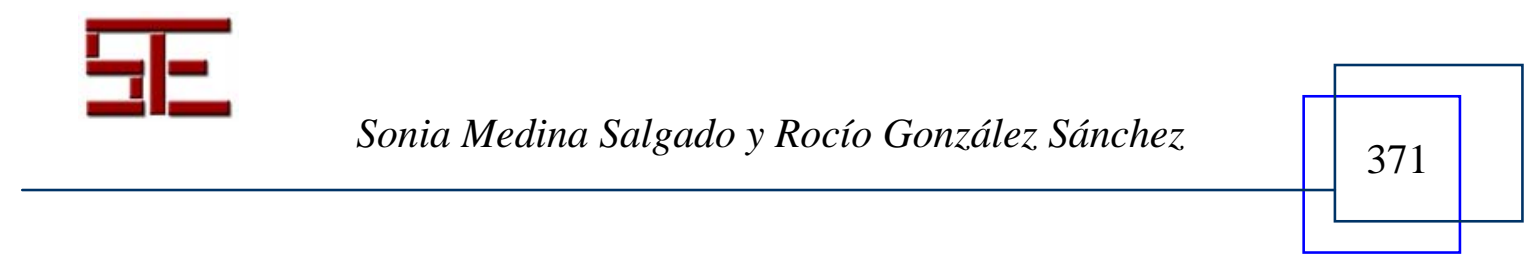




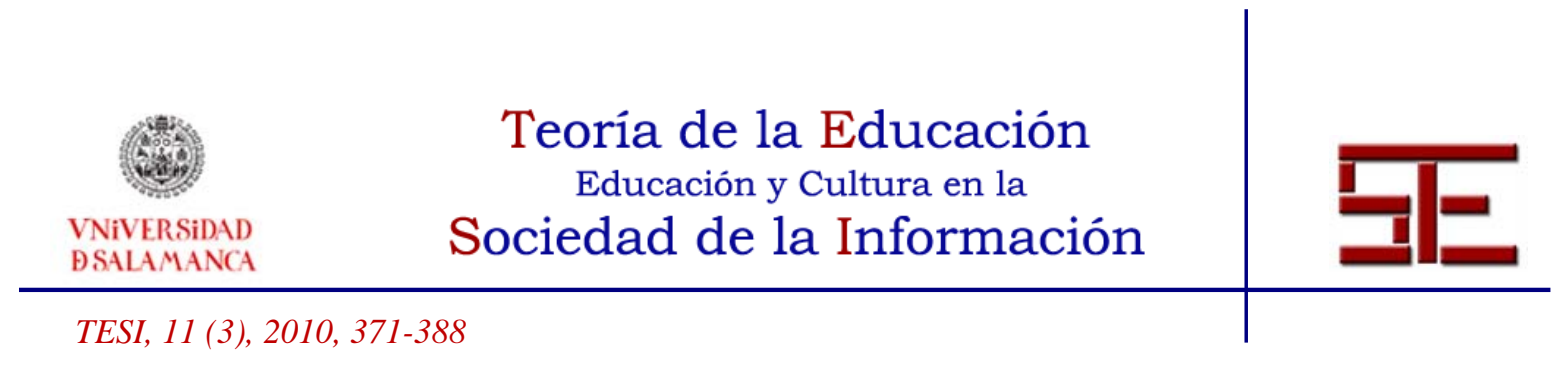

\title{
THE INFORMATION AND COMMUNICATION THECNOLOGIES (ICT) TRAINING IN TOURISM DIPLOMA: IS SUITABLE?
}

\begin{abstract}
:
This study analyses to what extent the knowledge acquired in ICT field fits the requirements asked for the tourism sector companies. First-year tourism students become familiar with the use of many ICT devices but they do not have sufficient background for using it properly in a professional context. In addition, the reality of the higher education in tourism studies reflects an apparent need for a greater number of credits for ICT knowledge and a greater number of places to study it.

Hence, our aim is to evaluate the gap between the level- and the kind- of ICT knowledge acquired and the labour markets required one. For that purpose, we carried out a three-phase empirical study, based on the assessments of students and the enterprises where they do their "practicum" course. The results support the need to optimize computing and information management courses content in order to meet the specific needs of the companies. Finally, some recommendations arise for helping professors to optimize the class work.
\end{abstract}

Keywords:

ICT competences, Tourism Diplomas, Practicum, European Higher Education Area

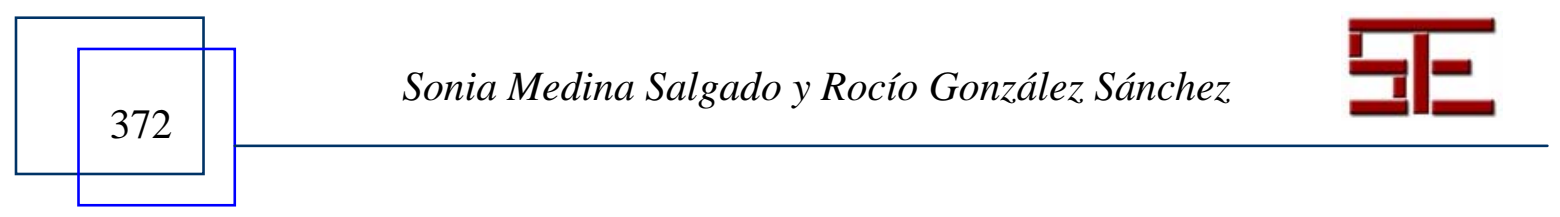




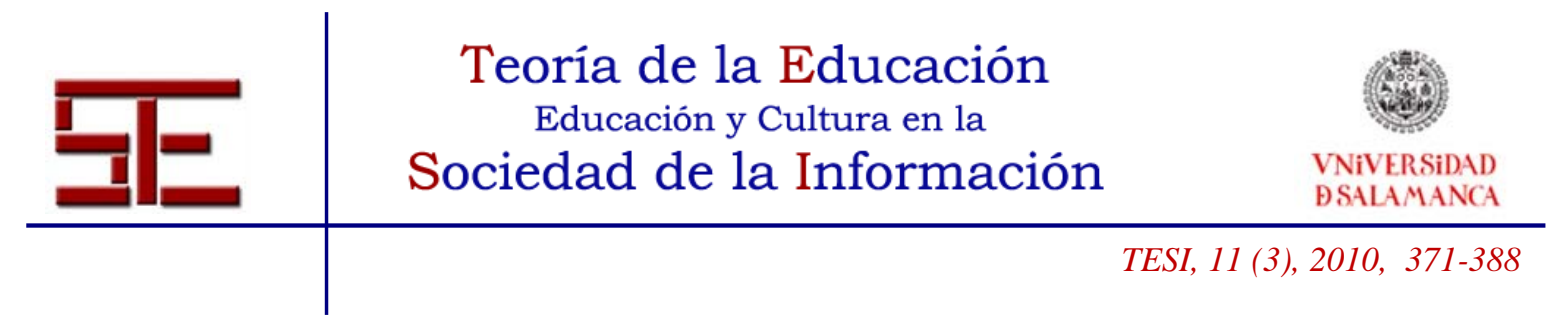

\section{LA FORMACIÓN EN TECNOLOGÍAS DE LA INFORMACIÓN Y DE LAS COMUNICACIONES (TIC) EN LA TITULACIÓN DE TURISMO: ¿ES ADECUADA?}

Fecha de recepción: 10/12/2009; fecha de aceptación: 16/01/2010; fecha de publicación: 28/02/10

Sonia Medina Salgado

sonia.medina@urjc.es

Universidad Rey Juan Carlos (Madrid).

Rocío González Sánchez

rocio.gonzalez@urjc.es

Universidad Rey Juan Carlos (Madrid).

\section{1.- INTRODUCCIÓN.}

El crédito europeo (ECTS) ${ }^{[1]}$ considera tanto el trabajo presencial como no presencial del alumno, lo que hace necesario un replanteamiento hacia nuevos escenarios pedagógicos, donde las metodologías y las herramientas a utilizar propicien el aprendizaje autónomo y reflexivo. De esta manera, se pretende lograr la formación integral del alumno a través de la adquisición de competencias que estimulen su creatividad (González Sánchez y García Muiña, 2008).

Sin embargo, este enfoque más teórico debe compatibilizarse con una necesidad de preparación práctica del futuro profesional que le haga atractivo en el mundo laboral, ya que en los 240 créditos que componen los planes de los títulos de grado, las competencias profesionales ocupan un lugar destacado. Numerosos estudios propugnan un enfoque más práctico y activo de docencia y destacan la notable preocupación de la comunidad educativa por las limitaciones a las que se enfrentan los profesores a la hora de integrar las diversas metodologías y la adaptación de las heterogéneas herramientas en los programas de enseñanza tradicional (Perry y Talley, 2001; Sprague et al., 1998).

Entre estas nuevas herramientas juega un papel importante las nuevas Tecnologías de la Información y las Comunicaciones (de ahora en adelante TIC). Las TIC hacen posible la

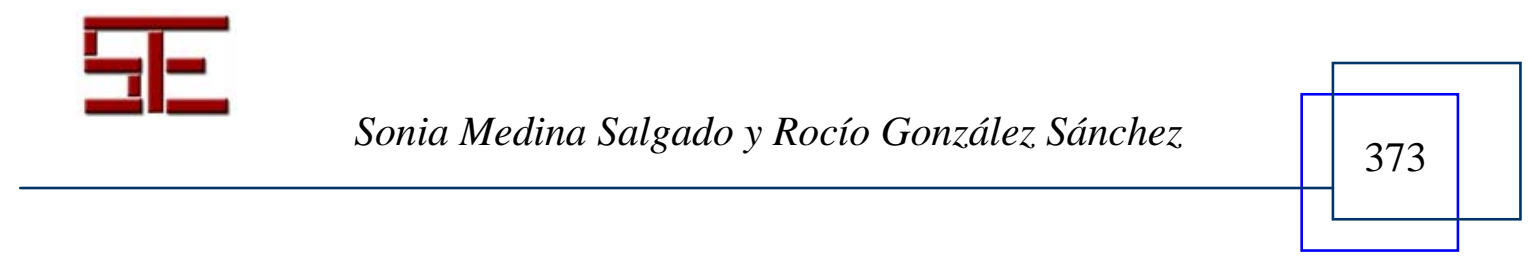




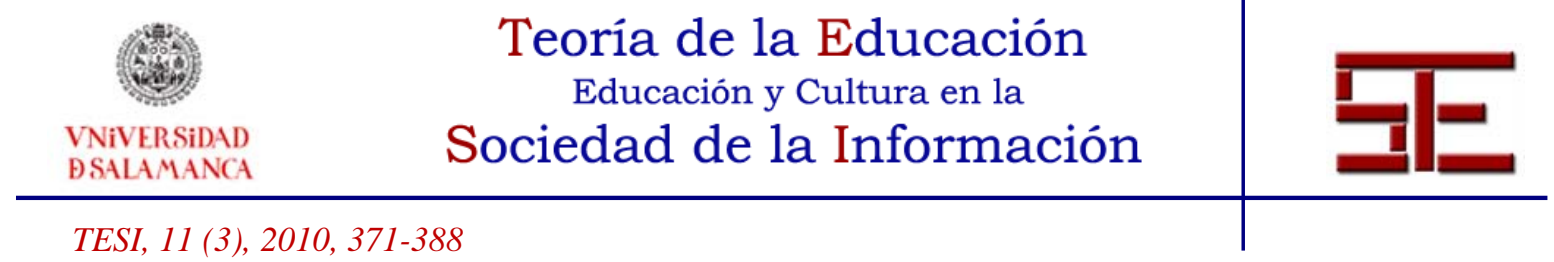

aplicación de métodos docentes más dinámicos y participativos y mejoran la calidad de la enseñanza universitaria (Laurillard, 1993, 2000; Santoveña Casal, 2007). De esta forma, los instrumentos que proporcionan permiten cubrir ciertas necesidades que surgen en el Espacio Europeo de Educación Superior.

\section{2.- EL CAPITAL HUMANO EN EL SECTOR DEL TURISMO.}

Utilizando la visión de Sáez Cala $(2006,23)$ definimos el turismo como "una actividad cultural emergente y un producto de modernidad". Dentro de las características que le son propias podemos destacar la naturaleza diversa, una estructura compleja y un dinamismo evolutivo (Pulido Fernández, 2008). El producto turístico se renueva constantemente, buscando nuevas oportunidades de satisfacer al turista. De esta búsqueda continua, se deriva el papel fundamental que juega en el sector turístico la innovación (Libro Blanco Título Grado de Turismo, 2005; Monfort et al, 1996). La innovación ha sido el origen de transformaciones de carácter, no sólo general, sino también estructural, lo que nos obliga analizar estas nuevas circunstancias tanto desde el lado de la oferta como de la demanda.

Desde el lado de la demanda, se rompen fronteras geográficas y en el número de operaciones realizadas, incrementado de forma significativa la oferta, alcanzando una distribución y comunicación de servicios global. Por el lado de la demanda, los actuales sistemas de distribución e información también han cambiado las reglas del juego, ya que permiten a los clientes acceder a una oferta mucho más amplia y con una información fácilmente comparable. Este nuevo turista desea un servicio personalizado y desarrolla nuevos hábitos de consumo más exigentes.

Este nuevo planteamiento obliga al sector a "replantear sus valores o normas de comportamiento y la manera en la cual educa a su fuerza de trabajo" (Buhalis, 1998, 411). Por lo tanto, para afrontar esta innovación los empleadores requieren de dos factores fundamentales: unas herramientas tecnológicas que le permitan el desarrollo de su trabajo de forma colaborativa y global dentro de la industria y un capital humano con unas importantes competencias profesionales en TIC y lingüísticas (Schnell, 2000; García Manjón y Pérez López, 2008).

Dragonetti y Roos (1998) definen el capital humano como el conjunto de recursos intangibles que poseen los miembros de las empresas. Este capital englobaría los conocimientos, habilidades y capacidades de las personas implicadas en la organización. En un sector como el del turismo, donde la experiencia del cliente es fundamental para retenerlo, resulta fundamental un capital humano productivo y con potencial. Su estudio y el desarrollo de prácticas, que mejoren tanto su generación como su explotación, resulta vital para la supervivencia de las organizaciones turísticas.

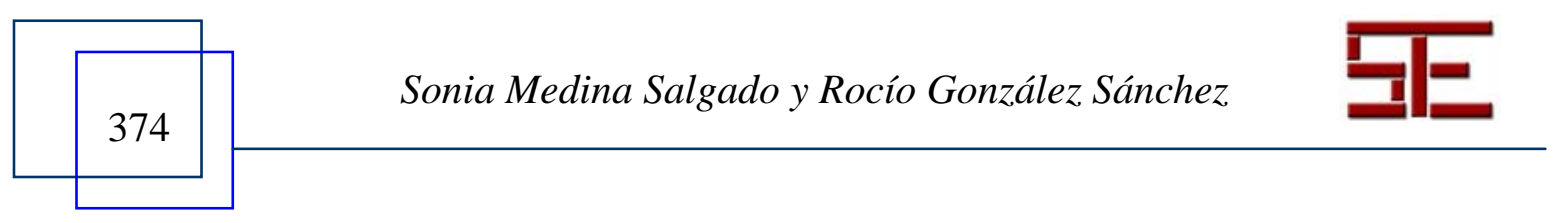




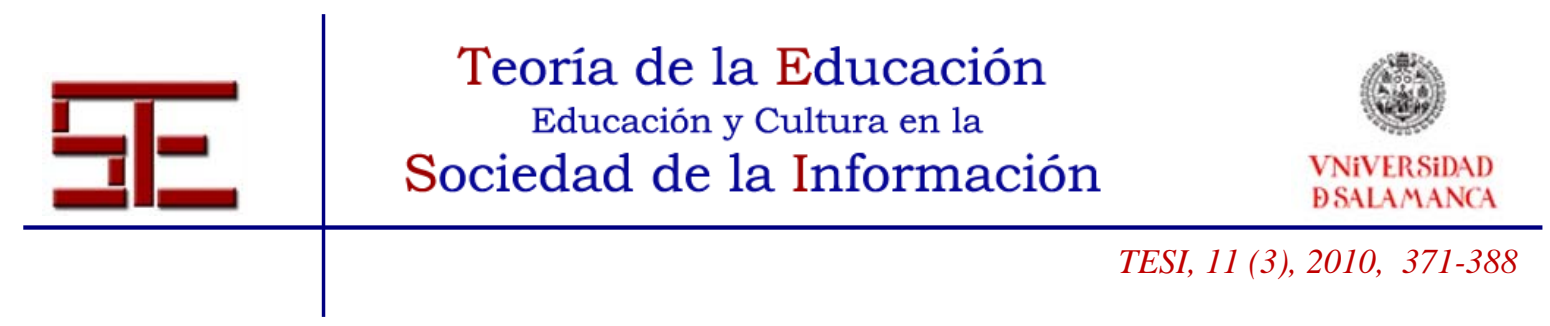

Uno de los retos fundamentales a los que se enfrentan los profesionales del turismo es la necesidad de adaptación continua a los cambios debido al dinamismo de la industria (Lobo Montero, 2007). A través de la formación de los trabajadores de la industria turística se mejora su capacidad de adaptación y, por lo tanto, se incrementará la flexibilidad e interactividad de los procesos productivos, que resultan más complejos debidos al entorno más competitivo y global al que se enfrentan (Buhalis, 1998; Pulido San Román et al., 1999).

Adicionalmente al proceso de Bolonia, se producen cambios legislativos y laborales para los profesionales del sector que les obligarán a enfrentan a nuevos retos que pretenden mejorar su formación. Un ejemplo claro, es la entrada en el 2010 del nuevo convenio colectivo estatal de las agencias de viajes que convierte a la formación en uno de los criterios claves para ascender de categoría profesional.

\subsection{Las competencias profesionales en el sector del turismo: El papel de las nuevas} tecnologías en la titulación de Turismo

El trabajo de Fernández (2005, 3) define las competencias como "cualquier característica individual que se pueda medir de un modo fiable, y que se pueda demostrar que diferencia de una manera significativa entre los trabajadores que mantienen un desempeño excelente de los adecuados o entre los trabajadores eficaces e ineficientes”. La mejora en la formación del profesional del sector permitirá el incremento en la calidad del servicio e información que se ofrece al cliente (Fernández et al., 2002). Además, esta formación en las competencias necesarias se convierte en un criterio determinante para el desarrollo de la carrera profesional del trabajador del sector.

Las nuevas tecnologías proporcionan herramientas que permiten acercar al alumno a situaciones reales, antes o incluso sin realizar prácticas en empresas (Fernández et al., 2002). Los alumnos que acceden a la titulación de Turismo están familiarizados con el uso de muchos dispositivos de TIC pero no tienen los conocimientos suficientes para un adecuado manejo en un ámbito profesional. El estudio de Guereño, et. al. (2008) pone de manifiesto que, aunque la incorporación de las TIC a la formación turística redunda en la mejora de la competitividad, existen todavía carencias en la incorporación de estos contenidos en las Facultades y en concreto en los estudios de Posgrado. Estos resultados se ven respaldados por otros trabajos, destacando el de Majó i Fernández (2004) que, tras analizar los antiguos planes de estudios de Diplomado en Turismo en 70 centros españoles, obtuvo un pobre resultado en cuanto a la obligatoriedad de las asignaturas con temática informática. La necesidad de incorporar estos contenidos se ha revelado como clave para el desarrollo de los nuevos estudios turísticos. Así, el nuevo Título de Grado en Turismo, elaborado por la ANECA, incluye una competencia específica relacionada con el aprendizaje en las Tecnologías de la Información y las






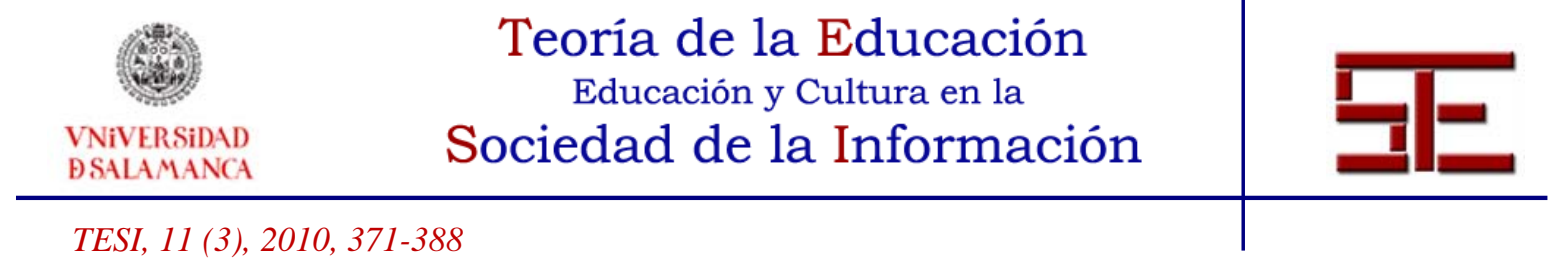

Comunicaciones. Con el fin de alcanzar esta competencia, el Libro Blanco incluye un listado de los principales conocimientos tanto disciplinares (saber) como profesionales (saber hacer) asociados al análisis y utilización de las TIC,s en los distintos ámbitos del sector turístico (ver tabla 1). La correcta estructuración de estos contenidos condicionará el éxito de la titulación a la hora de cubrir las necesidades discentes de los alumnos.

\begin{tabular}{|c|c|}
\hline \multicolumn{2}{|c|}{$\begin{array}{l}\text { 24. ANALIZAR Y UTILIZAR LAS TIC EN LOS DISTINTOS ÁMBITOS DEL SECTOR } \\
\text { TURÍSTICO }\end{array}$} \\
\hline \multicolumn{2}{|c|}{$\begin{array}{l}\text { Conocer las TIC como herramientas esenciales en la gestión, promoción y comercialización de las } \\
\text { empresas e instituciones del sector turístico, tanto a nivel interno de la empresa como nivel externo }\end{array}$} \\
\hline Conocimientos disciplinares (saber) & Conocimientos profesionales (saber hacer) \\
\hline $\begin{array}{l}\text { - Conocer los principios básicos de las TIC } \\
\text { - Conocer el funcionamiento de los GRS/GDS } \\
\text { - Conocer las tecnologías aplicadas a la } \\
\text { promoción y comercialización turística (bases } \\
\text { de datos, DMS, análisis y diseño de sistemas } \\
\text { de información turística, diseño y promoción } \\
\text { de sitios webs turísticos) } \\
\text { - Conocer las tecnologías aplicadas a la } \\
\text { planificación turísticas (bases de datos } \\
\text { espaciales, GIS, GPS, ...) } \\
\text { - Conocer las tecnologías aplicadas al soporte de } \\
\text { decisiones (Data Mining, CRM, ...) }\end{array}$ & $\begin{array}{l}\text { - Manejar herramientas ofimáticas integradas } \\
\text { - Manejar programas de gestión turísticas } \\
\text { - Diseñar, manejar y consultar bases de datos } \\
\text { para la gestión y planificación turísticas } \\
\text { - Utilizar Internet y sus diferentes servicios } \\
\text { - Diseñar y promocionar sitios webs turísticos } \\
\text { - Manejar sistemas de información de reservas } \\
\text { (CRS) y sistemas globales de distribución } \\
\text { (GDS) } \\
\text { - Manejar herramientas informáticas de } \\
\text { análisis estadísticos } \\
\text { - Manejar herramientas informáticas asociadas } \\
\text { a los sistemas de información geográfica } \\
\text { aplicados al análisis y la planificación turística }\end{array}$ \\
\hline
\end{tabular}

Tabla 1. Desglose de la competencia relativa a TIC del Libro Blanco del Título de Grado de Turismo

De cara a un correcto desarrollo de la estructuración de esta competencia en el nuevo Título de Grado en Turismo y con el objetivo, a medio y largo plazo, de un proceso de mejora continua, el presente estudio plantea la necesidad de conocer la actual situación de los alumnos de la Diplomatura de Turismo en materia de nuevas tecnologías. Para ello, este trabajo evalúa el desfase, tanto en el nivel como en el tipo de conocimientos requeridos en el uso de las TIC, entre la formación que recibe el alumnado y las exigencias del mercado laboral al que se enfrentaran los futuros profesionales. Para ello se ha realizado un estudio empírico en tres fases. La primera y segunda fase se ha dirigido a los alumnos de la Escuela de Turismo de la Universidad Rey Juan Carlos y la tercera se ha enfocado hacia las empresas en las que estos alumnos han desarrollado el denominado practicum.

En este punto, se hace necesario aclarar el papel que la asignatura del practicum tiene en el actual Plan de Estudios de la Diplomatura de Turismo de la Universidad Rey Juan

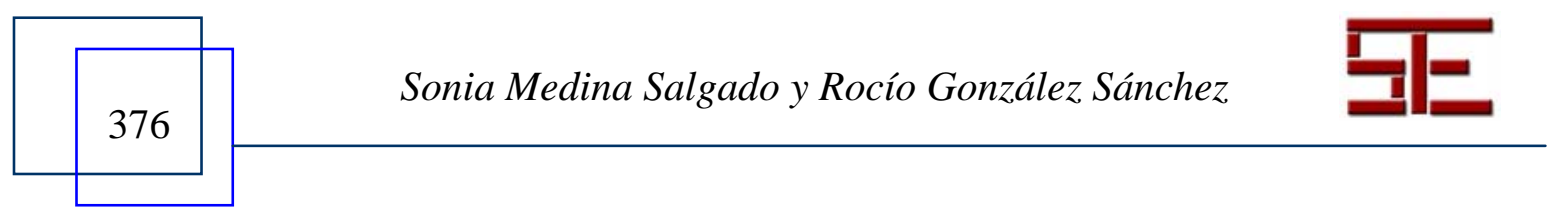




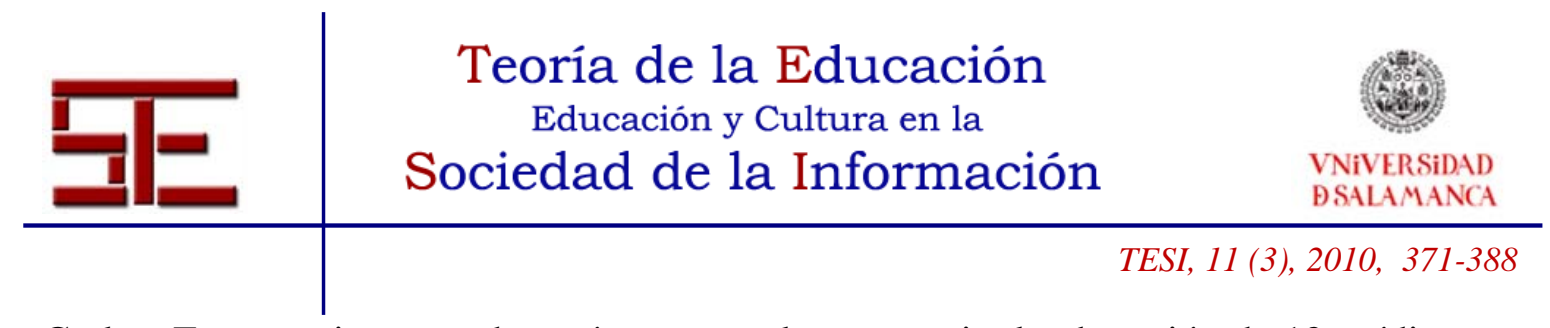

Carlos. Es una asignatura de carácter troncal que permite la obtención de 12 créditos en el tercer curso de la diplomatura de Turismo. Consiste en la realización de prácticas en empresas e instituciones del sector turístico con las que la mencionada Universidad ha establecido convenios de colaboración. El objetivo general de esta asignatura es la toma de contacto y familiarización por parte del alumno con los aspectos prácticos y habilidades que ha ido adquiriendo a lo largo de la carrera, y así facilitar su integración en el mundo laboral. (Guía del practicum de Turismo 2008-2009).

\section{3.- METODOLOGÍA.}

Para testar el desfase en el tipo y nivel de conocimientos requeridos en el uso de las TIC se realizaron dos tipos de cuestionarios. Uno dirigido a alumnos al comienzo y al final del segundo semestre y otro, a empresas en las que los alumnos habían realizado el practicum. Este último, nos ha permitido tener un primer conocimiento, de forma directa, de la aplicación práctica de los conocimientos disciplinares y profesionales en TIC adquiridos en las asignaturas cursadas. Incluir a las empresas del practicum, cuando los alumnos aún no han abandonado la Universidad, nos ha brindado la posibilidad de salvar una importante limitación en este tipo de estudios: la pérdida del contacto con el alumno que accede al mercado laboral y cuyos destinos profesionales son difíciles de averiguar.

Los alumnos sometidos a la evaluación fueron estudiantes universitarios de la Escuela de Turismo de la Universidad Rey Juan Carlos que cursaron, durante el segundo semestre del curso 2008-2009, asignaturas optativas y de libre elección relacionadas con las TIC en el sector turístico. En concreto, Informática de Gestión y Distribución en Touroperadores y Agencias de Viajes, Informática de Gestión y Distribución de transportes turísticos y Amadeus Selling Platform. En todas ellas, impartían o habían impartido docencia los profesores implicados en la investigación.

Se decidió llevar a cabo las experiencias en dos momentos diferentes del tiempo, uno previo a cursar las mencionadas asignaturas y otro con posterioridad a la realización de la docencia con el objeto de evaluar también, en un trabajo posterior, la percepción del alumnado sobre la utilidad y asimilación de los contenidos impartidos.

En la tabla 2 figuran los sujetos sometidos al análisis y de los cuales se han obtenido los datos. También figuran las variables que se han considerado para el estudio así como las distintas dimensiones con las que se han medido. Con las dos primeras variables se determina el impacto que el acceso y uso que hacen los alumnos tiene sobre su formación e interés por las TIC. Además estas variables permitir considerar algunas faltas de recursos que limitan el uso y la formación en TIC.

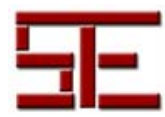

Sonia Medina Salgado y Rocío González Sánchez 




Para profundizar en la evaluación en el desfase de conocimientos se les pedía a los alumnos que expresaran sus apreciaciones respecto al dominio, relación y capacidades en TIC tanto al comienzo de la asignatura (datos pre) como al finalizar la misma (datos post). Estos datos junto a las valoraciones que los mismos alumnos hicieran de las prácticas realizadas en las empresas, podían reflejar la situación en cuanto a tipos de conocimientos y habilidades adquiridas y puestas en práctica por los alumnos.

Por último, las entrevistas telefónicas a las empresas iban dirigidas a recabar información acerca del trabajo desarrollado por los alumnos a lo largo de se estancia en ellas: valoración general, valoración del uso de las TIC, falta de formación, etc.

\begin{tabular}{|l|l|l|}
\hline $\begin{array}{l}\text { Sujetos de } \\
\text { análisis }\end{array}$ & Variables objeto de análisis & Dimensiones de análisis \\
\hline \multirow{3}{*}{ Alumnos } & $\begin{array}{l}\text { Acceso y uso en centro } \\
\text { educativo }\end{array}$ & $\begin{array}{l}\text { Disponibilidad de recursos en la Universidad } \\
\text { Frecuencia/tipo de empleo en la Universidad }\end{array}$ \\
\cline { 2 - 3 } & Acceso y uso en domicilio & $\begin{array}{l}\text { Disponibilidad de recursos en el domicilio } \\
\text { Frecuencia/tipo de empleo en el domicilio }\end{array}$ \\
\cline { 2 - 2 } & Familiarización y uso de las TIC & $\begin{array}{l}\text { Dominio de las TIC (pre y post) } \\
\text { Relación alumno/TIC } \\
\text { Capacidades con relación a las TIC (pre y post) }\end{array}$ \\
\cline { 2 - 3 } & Evaluación del practicum & $\begin{array}{l}\text { Satisfacción con las practicas realizadas } \\
\text { Utilidad de las practicas con relación a las TIC } \\
\text { Formación complementaria en las practicas }\end{array}$ \\
\hline
\end{tabular}

Tabla 2. Variables y dimensiones analizadas

La tabla 3 presenta la ficha técnica del estudio empírico. En ella se recoge la información desglosada por sujetos de análisis. Se agrupan los alumnos considerando la asignatura cursada. La técnica estadística utilizada fue el análisis estadístico descriptivo utilizando para el muestreo entrevista o cuestionario, según el sujeto a analizar.

\begin{tabular}{|c|c|c|c|c|}
\hline & $\begin{array}{c}\text { Empresas en las } \\
\text { que los } \\
\text { alumnos han } \\
\text { cursado el } \\
\text { practicum }\end{array}$ & $\begin{array}{c}\text { Informática de } \\
\text { Gestión y } \\
\text { Distribución en } \\
\text { Touroperadores y } \\
\text { Agencias de } \\
\text { Viajes }\end{array}$ & $\begin{array}{c}\text { Informática de } \\
\text { Gestión y } \\
\text { Distribución de } \\
\text { transportes } \\
\text { turísticos }\end{array}$ & $\begin{array}{c}\text { Amadeus } \\
\text { Selling } \\
\text { Platform }\end{array}$ \\
\hline Población & & 44 alumnos & 26 alumnos & 37 alumnos \\
\hline Muestra & & 26 alumnos & 7 alumnos & 23 alumnos \\
\hline$\underset{\text { respuesta }}{\text { Tasa }} d e$ & & $59,09 \%$ & $27 \%$ & $62,16 \%$ \\
\hline Toma de datos & \multicolumn{2}{|c|}{ Entrevista telefónica } & \multicolumn{2}{|c|}{ Cuestionario } \\
\hline
\end{tabular}

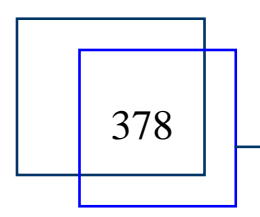




\begin{tabular}{|c|c|c|c|}
\hline  & $\begin{array}{r}\text { Teoria de la Educ } \\
\text { Educación y Cultura } \\
\text { Sociedad de la Info }\end{array}$ & $\begin{array}{l}\text { ación } \\
\text { la }\end{array}$ & $\begin{array}{l}\text { A } \\
\text { VNiVERSiDAD } \\
\text { BSALAMANCA }\end{array}$ \\
\hline & \multicolumn{3}{|c|}{ TESI, 11 (3), 2010, 371-388 } \\
\hline Muestreo & Las entrevistas se realizaron & \multicolumn{2}{|c|}{$\begin{array}{l}\text { El cuestionario se proporciono a los } \\
\text { alumnos en dos fases: en la fase del } \\
\text { inicio del curso y en la fase final del } \\
\text { curso. }\end{array}$} \\
\hline Ámbito de estudio & Sector turístico & \multicolumn{2}{|c|}{ Universidad Rey Juan Carlos } \\
\hline Periodo de análisis & \multicolumn{3}{|c|}{ Curso 2008-2009 } \\
\hline Unidad muestral & \begin{tabular}{|c|} 
Empresas colaboradoras del \\
practicum de la Escuela de \\
Turismo
\end{tabular} & \multicolumn{2}{|c|}{$\begin{array}{l}\text { Asignaturas del Departamento de } \\
\text { Economía de la Empresa (ADO) }\end{array}$} \\
\hline Fecha realización & Junio 2009 & \multicolumn{2}{|c|}{ Segundo semestre 2009} \\
\hline Técnica estadística & \multicolumn{3}{|c|}{ Análisis estadístico descriptivo } \\
\hline
\end{tabular}

Tabla 3. Ficha técnica del estudio empírico

La tasa de respuesta ha dado lugar a un total de 56 cuestionarios completos, es decir, que han respondido a las dos primeras fases del estudio. La tercera fase está condicionada a la realización del practicum con anterioridad al periodo estival.

\section{4.- RESULTADOS.}

4.1. El acceso de los alumnos y el uso que hacen de las TIC

\subsubsection{En la Universidad (tablas 4 y 5)}

Los alumnos consideran insuficiente la rapidez y la fiabilidad del acceso a Internet de que disponen en el centro universitario. Valorando con una media de 2,96 sobre 5 este acceso.

Mejorarían también la disposición de un puesto y un equipo adecuado obteniendo sobre este concepto una media de 3,33.

\begin{tabular}{|c|c|c|c|c|c|c|c|}
\hline & 1 & 2 & 3 & 4 & 5 & MEDIA & MODA \\
\hline Disponibilidad suficiente & 5 & 12 & 16 & 19 & 4 & 3,09 & 4 \\
\hline Acceso a Internet rápido y fiable & 6 & 14 & 14 & 16 & 4 & 2,96 & 4 \\
\hline $\begin{array}{l}\text { Disposición de puesto y equipo } \\
\text { adecuado }\end{array}$ & 4 & 10 & 13 & 20 & 8 & 3,33 & 4 \\
\hline
\end{tabular}

Tabla 4- Valoración de la disponibilidad de recursos en la Universidad

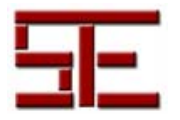




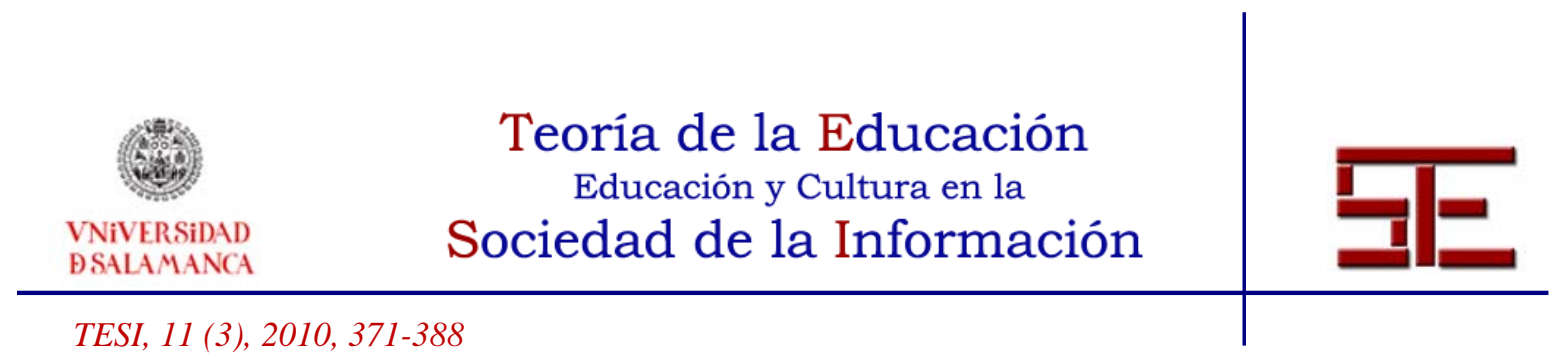

Los alumnos utilizan de forma mayoritaria los equipos informáticos en la Universidad para la realización de trabajos académicos y en grupo. También desarrollan aprendizaje colaborativo vía Internet y actividades con las distintas herramientas ofimáticas tales como procesadores de texto y software de presentaciones.

En el otro extremo, nos encontramos con el escaso uso de las herramientas que ofrece la Universidad para descargar software o de programas de diseño. Esto puede estar motivado, principalmente, por los mecanismos de seguridad de los sistemas de la Universidad que impiden la descarga de software en general y por que en la titulación de Turismo no han sido necesarias, hasta ahora, las herramientas de diseño para la formación.

\begin{tabular}{|c|c|c|c|c|c|c|c|}
\hline Uso para: & 1 & 2 & 3 & 4 & 5 & MEDIA & MODA \\
\hline Procesar textos & 1 & 5 & 16 & 16 & 16 & 3,76 & 5 \\
\hline Internet & 7 & 10 & 10 & 14 & 13 & 3,3 & 4 \\
\hline Trabajo académico & 1 & 2 & 6 & 12 & 32 & 4,36 & 5 \\
\hline $\begin{array}{l}\text { Comunicarse con otros } \\
\text { alumnos }\end{array}$ & 5 & 12 & 8 & 11 & 17 & 3,43 & 5 \\
\hline Descargar Software & 29 & 10 & 6 & 3 & 3 & 1,84 & 1 \\
\hline $\begin{array}{l}\text { Realización trabajos en } \\
\text { grupo }\end{array}$ & 2 & 3 & 6 & 13 & 29 & 4,21 & 5 \\
\hline Como apoyo a las clases & 4 & 6 & 14 & 15 & 13 & 3,52 & 4 \\
\hline Diseño & 17 & 11 & 16 & 4 & 3 & 2,31 & 1 \\
\hline Hojas de cálculo & 2 & 10 & 20 & 17 & 4 & 3,21 & 3 \\
\hline Presentaciones & 2 & 3 & 14 & 22 & 13 & 3,76 & 4 \\
\hline Colaboración via Internet & 2 & 6 & 9 & 18 & 17 & 3,81 & 4 \\
\hline Aprender en general & 3 & 8 & 16 & 18 & 9 & 3,41 & 4 \\
\hline Aprender en la titulación & 4 & 6 & 15 & 14 & 14 & 3,53 & 3 \\
\hline
\end{tabular}

Tabla 5- Valoración del uso de recursos en la Universidad

\subsubsection{En el domicilio (tablas 6 y 7 )}

La gran mayoría de los alumnos disponen de un equipo informático y lo pueden usar en su domicilio. También destaca la disponibilidad de Internet para una gran mayoría de alumnos (media de 4,66) lo que facilita el trabajo del docente para utilizar herramientas on-line, tal y como puede ser la plataforma de campus virtual. Por otro lado, es escasa la formación en TIC que el alumno desarrolla en casa con un exiguo 2,82 de media.

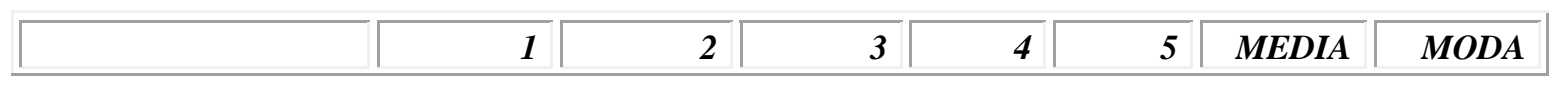

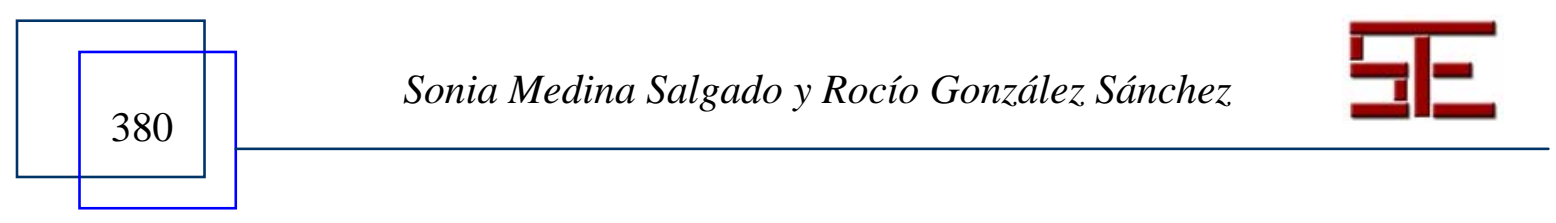




\begin{tabular}{l}
$\mid \begin{array}{l}\text { Teoría de la Educación } \\
\text { Educación y Cultura en la }\end{array}$ \\
\hline
\end{tabular}

Tabla 6- Valoración de la disponibilidad de recursos en el domicilio

En el domicilio sigue siendo mayoritario el uso del ordenador y la conexión a Internet para comunicarse con otros alumnos y para la realización de trabajo colaborativo y en grupo.

El desarrollo del trabajo académico a través de herramientas ofimáticas sigue siendo mayoritario. Sin embargo, cabe destacar el fuerte uso de los procesadores de texto y de herramientas de presentaciones en detrimento de las hojas de cálculo.

El alumno considera que el diseño y el aprendizaje, en general o de asignaturas de la titulación, son las actividades que menos realiza en los equipos domésticos.

\begin{tabular}{|c|c|c|c|c|c|c|c|}
\hline Uso para: & 1 & 2 & 3 & 4 & 5 & MEDIA & MODA \\
\hline Procesar textos & 1 & 2 & 5 & 25 & 22 & 4,18 & 4 \\
\hline Internet & 2 & 0 & 2 & 10 & 41 & 4,6 & 5 \\
\hline Trabajo académico & 2 & 0 & 4 & 17 & 32 & 4,4 & 5 \\
\hline Comunicarse con otros alumnos & 3 & 1 & 3 & 8 & 41 & 4,48 & 5 \\
\hline Descargar Software & 4 & 1 & 6 & 16 & 29 & 4,16 & 5 \\
\hline Realización trabajos en grupo & 3 & 0 & 2 & 15 & 34 & 4,43 & 5 \\
\hline Como apoyo a las clases & 3 & 3 & 9 & 18 & 23 & 3,98 & 5 \\
\hline Diseño & 12 & 15 & 14 & 8 & 4 & 2,57 & 2 \\
\hline Hojas de cálculo & 2 & 7 & 21 & 20 & 5 & 3,35 & 3 \\
\hline Presentaciones & 2 & 0 & 10 & 27 & 17 & 4,02 & 4 \\
\hline Colaboración via Internet & 3 & 0 & 0 & 25 & 25 & 4,3 & 5 \\
\hline Aprender en general & 1 & 9 & 11 & 23 & 11 & 3,62 & 4 \\
\hline Aprender en la titulación & 6 & 7 & 14 & 18 & 11 & 3,38 & 4 \\
\hline
\end{tabular}

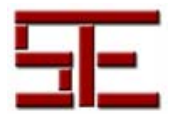




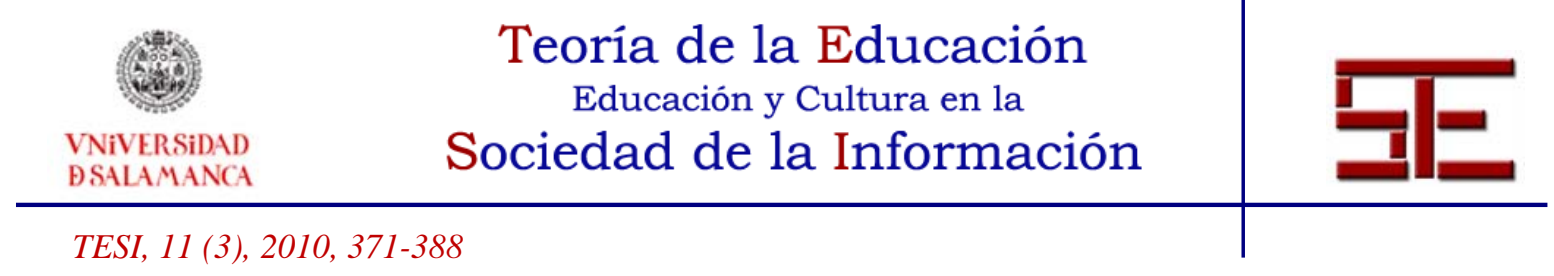

Tabla 7- Valoración del uso de recursos en el domicilio

4.2. La familiarización y las destrezas de los alumnos con relación a las TIC

\begin{tabular}{|l|r|r|r|r|}
\hline \multicolumn{1}{|c|}{} & \multicolumn{2}{|c|}{ PRE } & \multicolumn{2}{|c|}{ POST } \\
\hline \multicolumn{1}{|c|}{ MEDIA } & MODA & MEDIA & MODA \\
\hline Dominio técnico instrumental TIC alto & 3.182 & 3 & 4.093 & 5 \\
\hline Capacidad de diseño o producción de software & 2.018 & 1 & 2.464 & 3 \\
\hline
\end{tabular}

Tabla 8- Valoración de las capacidades previas y posteriores a cursar la asignatura

Los alumnos valoran de forma muy favorable el uso de las TIC en su formación y manifiestan un importante interés en alcanzar un mayor conocimiento instrumental de las mismas. Aunque no consideran que su uso se muy complicado, reconocen que no tienen el dominio adecuado de las distintas herramientas tecnológicas. Este hecho, puede verse motivado por la percepción que tienen de la necesidad de mayor formación en materia de TIC en su titulación universitaria.

\begin{tabular}{|c|c|c|c|c|c|c|c|}
\hline & 1 & 2 & 3 & 4 & 5 & MEDIA & MODA \\
\hline $\begin{array}{l}\text { Utilidad del uso de la } \\
\text { TIC en la formación } \\
\text { académica }\end{array}$ & 1 & 2 & 5 & 17 & 31 & 4,34 & 5 \\
\hline Interés en las TIC & 1 & 2 & 7 & 19 & 27 & 4,23 & 5 \\
\hline $\begin{array}{l}\text { Interés en formación } \\
\text { con TIC }\end{array}$ & 2 & 1 & 7 & 16 & 30 & 4,27 & 5 \\
\hline $\begin{array}{l}\text { Sencillez en el uso de } \\
\text { las TIC }\end{array}$ & 2 & 4 & 17 & 21 & 12 & 3,66 & 4 \\
\hline $\begin{array}{l}\text { Adecuado dominio de } \\
\text { las TIC }\end{array}$ & 6 & 16 & 21 & 11 & 2 & 2,77 & 3 \\
\hline $\begin{array}{l}\text { Mejor aprendizaje con } \\
\text { ordenador }\end{array}$ & 1 & 4 & 13 & 25 & 13 & 3,8 & 4 \\
\hline $\begin{array}{l}\text { Mejor rendimiento } \\
\text { académico gracias a las } \\
\text { TIC }\end{array}$ & 3 & 4 & 12 & 22 & 15 & 3,75 & 4 \\
\hline $\begin{array}{l}\text { Formación suficiente en } \\
\text { la Universidad en } \\
\text { materia de TIC }\end{array}$ & 9 & 11 & 17 & 17 & 1 & 2,82 & 4 \\
\hline
\end{tabular}

Tabla 9- Valoración de la relación con las TIC

El hecho de que los alumnos valoren de forma favorable o muy favorable sus capacidades previas a la realización de la asignatura, ha condicionado un menor

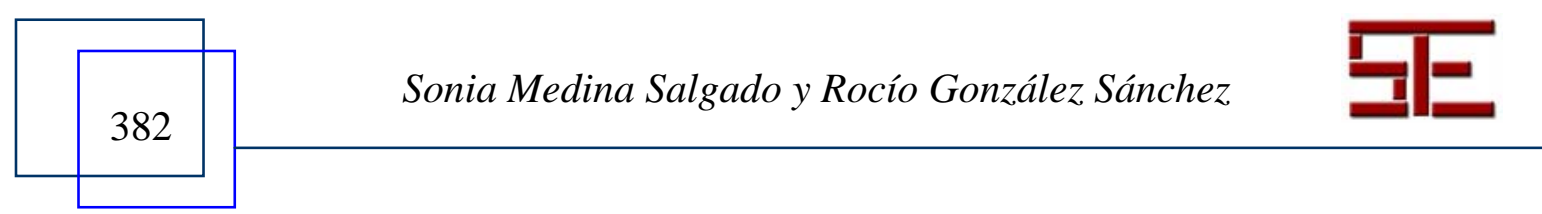




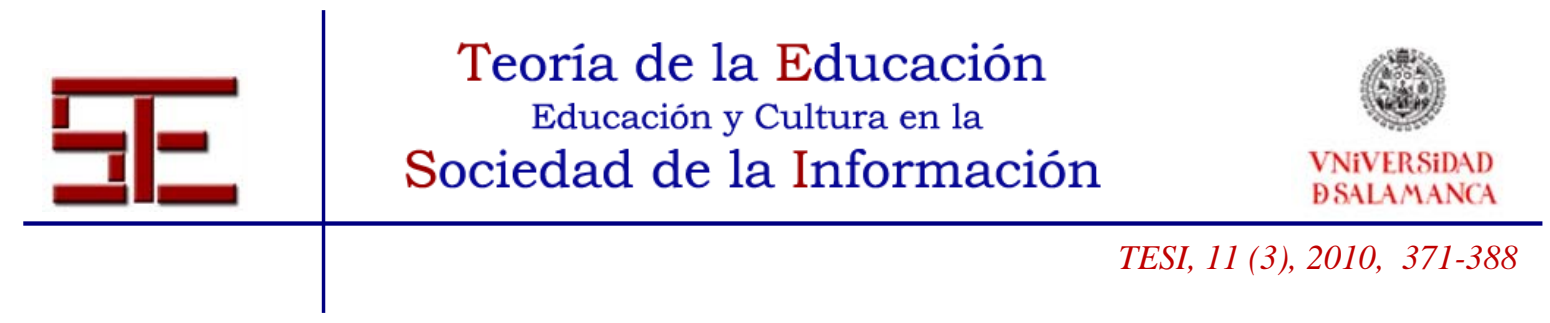

incremento de las mismas. Por otro lado, el hecho de no disponer de ciertas herramientas, tales como bases de datos y conexión a Internet, ha supuesto una penalización en las valoraciones de los alumnos respecto a su capacidad posterior.

\begin{tabular}{|l|r|r|r|r|}
\hline \multicolumn{1}{|c|}{} & \multicolumn{2}{|c|}{ PRE } & \multicolumn{2}{|c|}{ POST } \\
\hline Capacidad de uso de procesadores de texto & MEDIA & MODA & MEDIA & MODA \\
\hline Capacidad de trabajo con información digital & 4,23 & 5 & 4,29 & 5 \\
\hline Capacidad de uso Internet & 3,98 & 5 & 4,18 & 5 \\
\hline Capacidad de uso y recepción de correo & 4,5 & 5 & 4,41 & 5 \\
\hline Capacidad de uso de bases de datos & 4,59 & 5 & 4,43 & 5 \\
\hline Capacidad de uso Internet para comunicarse & 3,68 & 4 & 3,57 & 5 \\
\hline Capacidad uso hoja de cálculo & 4,46 & 5 & 4,32 & 5 \\
\hline Capacidad de elaboración de presentaciones multimedia & 4,14 & 4 & 4,18 & 5 \\
\hline Capacidad de elaboración de webs sencillas & 4,25 & 5 & 4,34 & 5 \\
\hline
\end{tabular}

Tabla 10- Desglose de las valoraciones de las capacidades previas y posteriores a cursar la asignatura

4.3. Evaluación del practicum: valoración de los alumnos y de las empresas implicadas

Los alumnos manifiestan una gran satisfacción con las prácticas realizadas en las distintas empresas, el hecho de poder elegir libremente la empresa en la que realizarlas, ha favorecido el correcto desarrollo de las mismas.

Esta satisfacción se traduce en un deseo generalizado de repetir las prácticas en la misma empresa y en la creencia del alumno de haber logrado que las empresas quedasen satisfechas con la formación genérica y en TIC del alumnado acogido en prácticas.

Aunque la valoración de la utilidad de la formación, tanto general como en TIC, es media seria recomendable un mayor ajuste, para lograr incrementar esta puntuación en sucesivos estudios.

Durante la realización de las prácticas se han utilizado de forma cotidiana distintas herramientas tecnológicas, destacando entre ellas las de comunicación, ofimática y software específico. La mayor parte de los alumnos manifiestan haber recibido formación complementaria en la realización de las practicas auque este porcentaje podría ser mejorable.

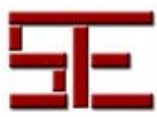






\begin{tabular}{|l|r|r|}
\hline & MEDIA & MODA \\
\hline Satisfacción general con las practicas & 4,36 & 5 \\
\hline Libertad en la elección de la empresa & & 5,79 \\
\hline Repetición de las practicas en la misma empresa & 4,14 & 5 \\
\hline Satisfacción de la empresa con la formación del alumno & 4,63 & 5 \\
\hline Satisfacción de la empresa con la formación en TIC del alumno & 4,41 & 5 \\
\hline Utilidad de la formación previa para las prácticas & 3,59 & \\
\hline Utilidad de los programas informáticos de la asignatura para las practicas & 3,43 & 4 \\
\hline Utilidad de los programas informáticos de otras asignaturas para las practicas & 3,11 & 3 \\
\hline Relación tareas de las practicas con la formación universitaria & 3,61 & 4 \\
\hline Utilización cotidiana de las TIC en la labor desarrollada & 4,54 & 5 \\
\hline Utilización cotidiana de herramientas de comunicación en la labor desarrollada & 4,52 & 5 \\
\hline Utilización cotidiana de herramientas de ofimática & 4,48 & 5 \\
\hline Utilización cotidiana de herramientas de software específico & 4,14 & 5 \\
\hline Utilización cotidiana de Intranet de la empresa & 3,76 & 5 \\
\hline Formación complementaria sobre TIC en las practicas & 3,86 & 5 \\
\hline Formación complementaria en otras áreas en las practicas & 3,9 & 5 \\
\hline
\end{tabular}

Tabla 11- Valoraciones de alumnos sobre el practicum

Se han entrevistado a las 12 empresas en las que los alumnos de las asignaturas objeto de estudio han realizado el practicum. Estas empresas pertenecen, en su mayoría, al ámbito de la intermediación, principalmente agencias de viajes minoristas y agencias de viajes mayoristas-minoristas. También contamos en la muestra con empresas del ámbito del alojamiento, coincidiendo, en su mayoría, con hoteles de 4 a 5 estrellas.

La valoración media que hacen las empresas de la formación general de los alumnos en prácticas, alcanza un 4,5 sobre 5 . Una puntuación realmente alta, que manifiesta la satisfacción de las empresas con la labor realizada por el alumnado. Sin embargo, esta valoración, aún siendo satisfactoria, disminuye en el caso de las nuevas tecnologías que no llega al 4. El motivo principal de esta menor valoración estriba en la necesidad de incrementar la formación en Sistemas Globales de Distribución (GDS) y en gestión de reserva hotelera.

Alguna de las empresas nos informa que llevan a cabo cursos de formación propia, aunque confirman que la mayor parte del aprendizaje de los alumnos en prácticas se

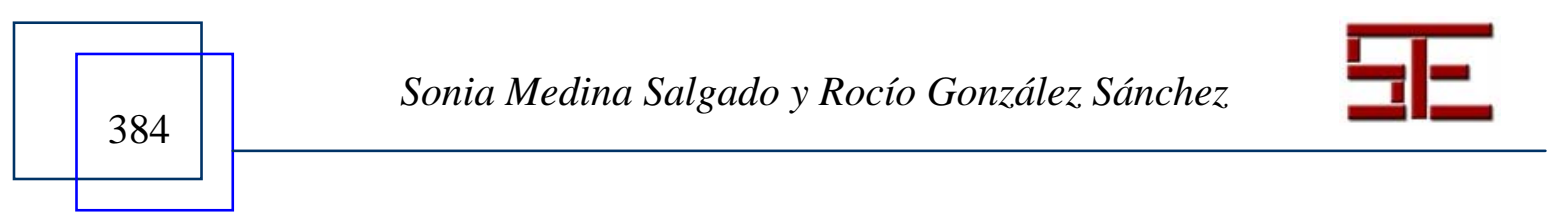




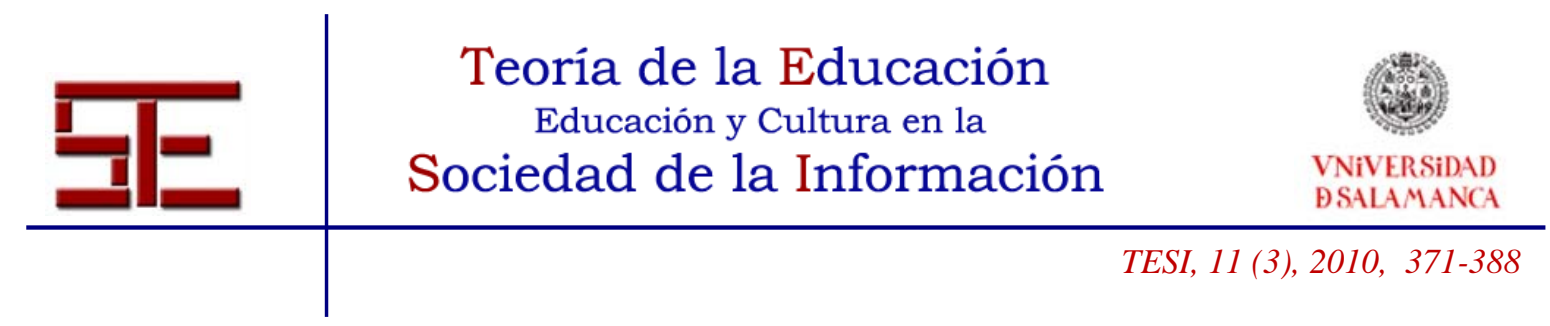

realiza durante el desarrollo de su labor diaria. La formación propia suele estar relacionada en su mayoría con los sistemas específicos que utilizan.

Destacan también la necesidad de mejorar otras capacidades tales como la identificación de destinos turísticos o la comunicación en una segunda o tercera lengua extranjera. Precisamente es la identificación de destinos turísticos a través de software especializado, uno de los contenidos recomendados por los alumnos en las observaciones adicionales de los cuestionarios.

Por último, cabe subrayar la importancia que las empresas otorgan a la positiva actitud e interés demostrados por los alumnos acogidos en practicas. Ello, en opinión de las empresas, ha facilitado un correcto y satisfactorio desarrollo de la actividad en prácticas. Esta valoración coincide con la percepción manifestada por los alumnos con relación a su valoración positiva de la actividad realizada. Igualmente, hay una importante coincidencia con el interés mostrado por los alumnos en profundizar en su aprendizaje en general y en el relacionado con las TIC en particular.

\section{5.- CONCLUSIONES.}

En el nuevo contexto económico centrado en la sociedad del conocimiento, las TIC, y en particular la proliferación del uso de Internet, desempeñan un papel fundamental en todos los sectores, en general, y en la industria turística en particular. De esta forma, el sector ha alcanzando una distribución y comunicación de servicios global. Las nuevas herramientas tecnológicas permiten a los clientes acceder a una oferta mucho más amplia y con una información fácilmente comparable. Este nuevo turista desea un servicio personalizado y desarrolla nuevos hábitos de consumo más exigentes.

Los objetivos del Grado en Turismo son definidos teniendo en cuenta estas nuevas características y la evolución de la actividad turística desarrollada en un contexto de rápida transformación y exigencias de calidad, competitividad y sostenibilidad y de una creciente internacionalización. En definitiva, se hace referencia a un nuevo modelo de conocimiento, que se adquiere a través de diversos medios, actividades y experiencias.

Los resultados apoyan una percepción muy positiva del uso de las TIC por parte de los alumnos que manifiestan un importante interés en continuar con su formación en esta materia a lo largo de su vida, tanto académica como laboral. En general, reconocen mejorar en sus capacidades relacionadas con las TIC tras cursar la asignatura. Sin embargo, el hecho de no disponer de ciertas herramientas, que consideran fundamentales en el desarrollo de la docencia en el aula, supone una penalización en las valoraciones de los alumnos respecto a su capacidad posterior.

El estudio del practicum, con la doble perspectiva del alumno y la empresa, nos ha permitido obtener interesantes resultados. En un elevado porcentaje los alumnos manifiestan una gran satisfacción con las prácticas realizadas en las distintas empresas,

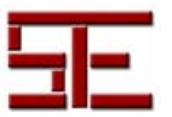




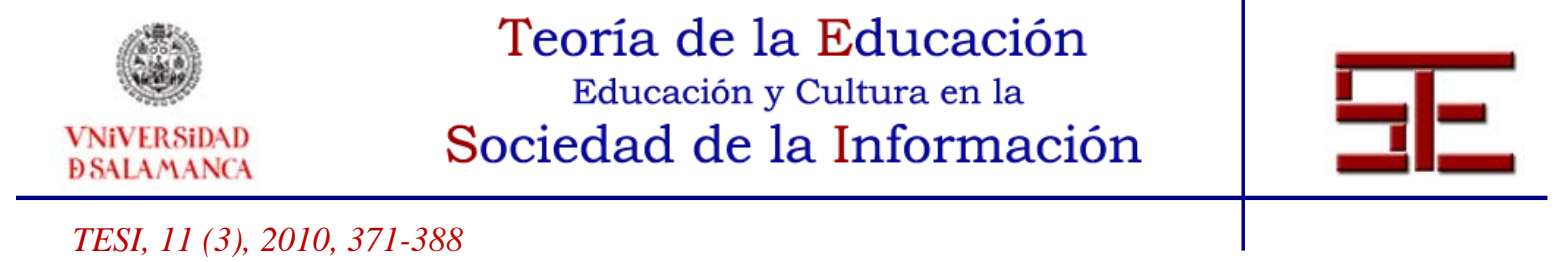

el hecho de poder elegir libremente la empresa en la que realizarlas, ha favorecido el correcto desarrollo de las mismas. Esta satisfacción se traduce en un deseo generalizado de repetir las prácticas en la misma empresa y en la creencia del alumno de que las empresas han quedado satisfechas con la formación genérica y en TIC del alumnado acogido en prácticas. La valoración media que hacen las empresas de la formación general de los alumnos en prácticas es considerablemente alta con la labor realizada por el alumnado. Sin embargo, aún existe recorrido en el incremento la formación en Sistemas Globales de Distribución (GDS) y en gestión de reserva hotelera.

Por otro lado, estas valoraciones, aún siendo muy positivas por ambos lados, demuestran que aún se consideran los estudios turísticos en España de un modo tradicional, como formación de nivel medio dirigida el ejercicio más ejecutivo que directivo en el sector. Ello hace que, como futura líneas de investigación, nos planteemos complementar el presente estudio con un conocimiento en profundidad de las necesidades en materia de TIC para puestos de dirección media y alta en el sector. También, estudiar mejor el tipo de puestos ejercidos actualmente en el sector por los alumnos que se licencian permitiría poder desarrollar mejor los contenidos para futuras asignaturas relacionadas con las TIC.

Además, incluir formación en TIC ligada al ejercicio de labores relacionadas con la planificación y gestión turística en las nuevas titulaciones, permitiría alcanzar muchos de los conocimientos que la propia ANECA reconoce en el Libro Blanco de la Titulación de Grado en Turismo, tales como, las tecnologías aplicadas al soporte de decisiones (Data Mining, CRM, ...). No en vano trabajos como el de García Manjón y Pérez López (2008) evidencian la necesidad de adquirir competencias en TIC ligadas a las de gestión y planificación turística así como al ejercicio del trabajo en inglés.

En definitiva, este estudio ha puesto de manifiesto la necesidad de una mentalidad de mejora continua que nos permitirá adaptarnos a una realidad cambiante y detectar posibles desviaciones para su rápida corrección. El permitir a los alumnos y a las empresas iniciar un debate abierto y sincero es apostar por el aprendizaje activo, dinámico, continuo y cercano a la realidad empresarial dentro del marco del Espacio Europeo de Educación Superior.

\section{6.- REFERENCIAS.}

Aneca (2005). Libro Blanco Título de Grado en Turismo.

Guereño Omil, B., Abad Galzacorta, M., Goytia Prat, A. y Alzua Sorzabal, A. (2008).

TIC y Postgrados en Turismo: un acercamiento al análisis de la oferta.

Buhalis, D. (1998). Strategic use of information technologies in the tourism industry.

Tourism Management, 5, 19, 409-421.Ponencia presentada en el VII Congreso

Turismo y Tecnología de la Información y las Comunicaciones. Turitec.

Drogonetti, R. (1998). Capital Intelectual. www.GestióndelConocimiento.com

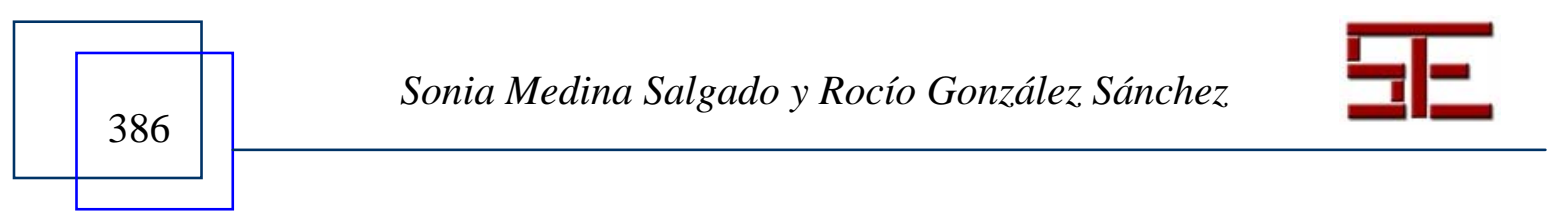




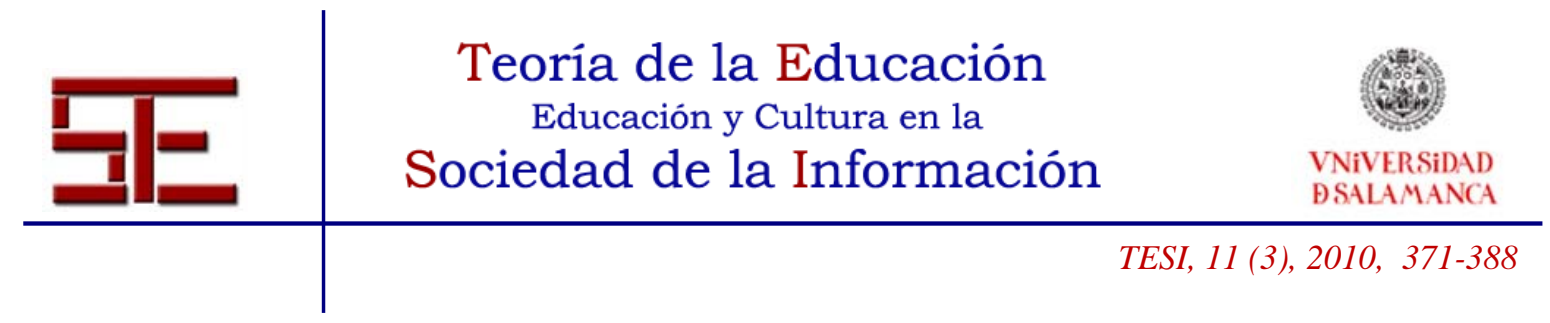

Escuela Universitaria de Turismo (2008-2009). Guía del practicum de Turismo, Subdirección de alumnos y practicum. Universidad Rey Juan Carlos.

Fernández, J. M. (2005). Matriz de competencias del docente de educación básica, Revista Iberoamericana de educación, 36-2. En http://www.rieoei.org/investigacion/939 Fernandez.PDF.

Fernández, E., Olmedo I. y Moratilla A. (2002). E-Learning: Hacia la próxima frontera del aprendizaje y el conocimiento en turismo. Ponencia presentada en el IV Congreso Turismo y Tecnología de la Información y las Comunicaciones. Turitec.

García Manjón, J. V. y Pérez López, M. C. (2008). El grado en Turismo: Un análisis de las competencias profesionales, Cuadernos de Turismo, 21, 67-83.

González Sánchez, R. y García Muiña, F. E. (2008). El blog en la docencia universitaria, ¿una herramienta útil para la convergencia europea?. Ponencia presentada en las VI Jornadas Internacionales de Innovación Universitaria. Universidad Europea de Madrid.

Laurillard, D. (1993). Rethinking university teaching: A framework for the effective use of educational technology. Londres, Routhledge.

- (2000). New technologies, students and the curriculum. The impact of communications and information tecnology on higher education, en SCOTT, P. (ed.) Higher Education Re-formed. Londres, Falmer Press, 133-153.

Lobo Montero, P. (2007). Posibilidades profesionales de los Diplomados de Turismo, Cuadernos de Turismo, 20, 131-151.

Majó i Fernández, J. (2004). Las tecnologías de la información y las comunicaciones en el currículum de los estudios de Turismo, V Congreso Turismo y Tecnología de la Información y las Comunicaciones. Turitec.

Perry, G. \& Talley, S. (2001). Online video case studies and teacher education: A new tool for pre-service teacher education, Journal of Computing in Teacher Education, 17 (4), 6-31.

Pulido Fernández, J. I. (2008). Delimitación conceptual y tipologías del turismo rural, en PULIDO FERNÁNDEZ. J. I. (coords). El turismo rural. Estructura económica y configuración territorial en España. Madrid: Síntesis, 21-49.

Pulido San Román, A., Sancho Pérez, A. y Maset Llaudes, A. (1999). La innovación en las empresas turísticas y las necesidades de formación, I Congreso Turismo y Tecnología de la Información y las Comunicaciones. Turitec.

Sáez Cala, A. (2006). La actividad turística: conceptos, clasificaciones y fuentes de información, En SÁEZ CALA, A., MARTÍN URBANO, P. y PULIDO FERNÁNDEZ, J. I. (coords). Estructura económica del turismo. Madrid: Síntesis, 21-53.

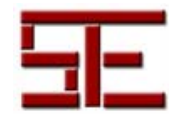




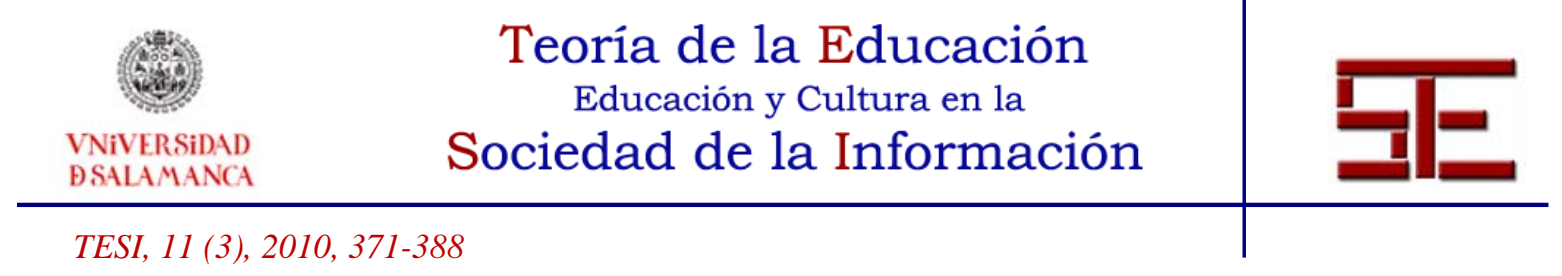

Santoveña Casal, S. M. (2007). Las nuevas tecnologías y la educación superior, Quaderns Digitals, 46, 1-13.

Schnell, K. (2000). Insights to the Development and Implementation of an Information System for Tourism,Environment and Soft Mobility, 40th Congreso of the Notas: European Regional Science Association, 1-14.

[1] European Credits Transfer System: Cuantifica en 60 créditos el volumen de trabajo total de un estudiante a tiempo completo durante un curso académico. Esto supondría unas 25 horas de trabajo por parte del estudiante a tiempo completo a la semana.

Para citar el presente artículo puede utilizar la siguiente referencia:

Medina Salgado, S. y González Sánchez, R.: (2010). "La formación en tecnologías de la información y de las comunicaciones (TIC) en la titulación de turismo: ¿Es adecuada?”. En De Pablos Pons, J. (Coord.) Buenas prácticas de enseñanza con TIC [monográfico en línea]. Revista Electrónica Teoría de la Educación: Educación y Cultura en la Sociedad de la Información. Vol. 11, $\mathrm{n}^{\circ} 1$. Universidad de Salamanca, pp. 371-388. [Fecha de consulta: $\mathrm{dd} / \mathrm{mm} / \mathrm{aaaa}$ ].

http://revistatesi.usal.es/ revistas_trabajo/index.php/revistatesi/article/view/6310/6323

ISSN: 1138-9737

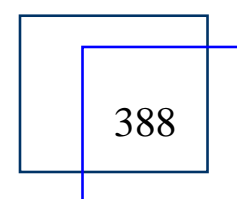

Sonia Medina Salgado y Rocío González Sánchez 